\title{
Respuesta de la pitahaya roja (Hylocereus undatus) a la aplicación de dos abonos orgánicos sólidos en la zona de San Carlos, Los Ríos, Ecuador
}

\author{
Response of the red pitahaya (Hylocereus undatus) to the application \\ of two solid organic fertilizers in the San Carlos Area, Los Ríos, Ecuador \\ Eduardo De la Cruz Sánchez ${ }^{1}$, Jéssica Morán Morán 2*, Rodrigo Cabrera Verdezoto ${ }^{3 *}$, \\ Cesar Cabrera Verdesoto², José Alcívar Cobeña ${ }^{2}$,Fabricio Meza Bone ${ }^{1}$
}

\begin{abstract}
RESUMEN
La búsqueda de nuevas alternativas en la fertilización del cultivo de pitahaya es relevante, ya que va en beneficio de la conservación del medioambiente y potenciar los rendimientos de este cultivo. El objetivo de este trabajo fue evaluar la respuesta de la pitahaya roja a la aplicación de dos abonos orgánicos sólidos en tres dosis de aplicación en las condiciones agroecológicas de la Parroquia San Carlos, del Cantón Quevedo, Ecuador. Se utilizaron 315 plantas del genero Hylocereus undatus, los factores en estudio fueron el humus y compost, en las dosis $8 ; 4$ y $2 \mathrm{t} \mathrm{ha}^{-1}$ Se utilizó un diseño de bloques completos al azar (DBCA) con siete tratamientos y tres repeticiones. Los abonos orgánicos mostraron un efecto similar cuando fueron utilizados en las mismas dosis; cada una de las variables evaluadas tuvo una tendencia a la disminución a medida que decreció la dosis de abono aplicado. La dosis que permitió maximizar el rendimiento en este cultivo en ambos tipos de abono fue la de $8 \mathrm{t} \mathrm{ha}^{-1}$.
\end{abstract}

Palabras claves: Compost, humus, rendimiento, fertilizantes orgánicos, suelo.

\section{ABSTRACT}

The search for new alternatives in the fertilization of the pitahaya crop is relevant, since it is in benefit of the conservation of the environment and to enhance the yields of this crop. The objective was to evaluate the response of the red pitahaya to the application of two solid organic fertilizers in three doses of application in the agroecological conditions of parish San Carlos, canton Quevedo, Ecuador. 315 plants of the genus Hylocereus undatus were used; the factors studied were humus and compost, in doses 8; 4 and $2 t \mathrm{ha}^{-1}$ A randomized complete block design (DBCA) with seven treatments and three replicates was used. Organic fertilizers showed a similar effect when used in the same doses; Each of the variables evaluated had a tendency to decrease as the applied fertilizer dose decreased. The dose that allowed to maximize the yield in this crop in both types of fertilizer was the one of 8 tha-1.

Key words: Compost, humus, organics fertilizers, yield, Soil.

\section{Introducción}

La pitahaya (Hylocereus undatus) es de la familia de las cactáceas y crece en las zonas tropicales (López et al., 2002). Su fruta tiene una corteza suave de intenso color rojo, cubriendo una carne jugosa de color claro con un sin número de semillas negras pequeñas (Montesinos et al., 2015). Este cultivo se ha establecido en la Provincia de
Los Ríos San Carlos, Mocache y el Empalme en la última década debido que tiene una gran demanda a nivel nacional e internacional por lo tanto se están buscando alternativas de fertilización que permitan obtener altos rendimientos y frutos saludables.

Por lo tanto, la fertilidad del suelo es uno de los temas más relevantes en la agricultura, ya que son pocos los suelos suficientemente fértiles para satisfacer las necesidades nutritivos del cultivo

1 Facultad de Ciencias Agrarias. Universidad Técnica Estatal de Quevedo (UTEQ). Ecuador.

2 Facultad de Ciencias Naturales y de la Agricultura. Carrera de Ingeniería Agropecuaria. Universidad Estatal del Sur de Manabí (UNESUM). Ecuador.

3 Instituto Técnico Superior Ciudad de Valencia. Ecuador.

* Autores por correspondencia: jessica.moran@unesum.edu.ec; ing.rcabrerav@gmail.com

Fecha de Recepción: 27 febrero, 2019.

Fecha de Aceptación: 1 septiembre, 2019. 
de pitahaya y le permita mejorar su rendimiento durante un período prolongado de tiempo (FAO, 1999). A causa de la necesidad apremiante de aumentar la producción de alimentos, se han elevado de forma considerable la producción y empleo de distintos abonos orgánicos como fuente de fertilización.

Pero las investigaciones a nivel nacional e internacional sobre nutrición en el cultivo de pitahaya son escasas. Por esto la fertilización que se emplea en la mayoría de las plantaciones de pitahaya se basa en experiencias propias de los productores (Ponce y Kodera 2003), siendo la fertilización química la que más se usa, sin tener un conocimiento técnico sobre la eficiencia y manejo de los fertilizantes. Esto trae consigo un grave deterioro en los suelos agrícolas lo que repercuten en la producción del cultivo.

Resultado de estos problemas, se ha observado un creciente interés en el uso de materiales orgánicos como fuente de fertilización para este cultivo, ya que constituyen una alternativa factible para proteger el recurso suelo y a su vez poder mantener rendimientos óptimos en el cultivo de pitahaya (Otiniano et al., 2006). Su uso trae consigo grandes ventajas como la reducción del daño ambiental o riesgos a la salud (Altieri y Rosset, 1995). Por ello se vio la necesidad de realizar esta investigación en el cultivo de pitahaya, donde se determine la fuente de fertilización orgánica que expresaron una mayor producción en este cultivo (Manani et al., 2012; Iguácel et al., 2010). El objetivo de esta investigación fue evaluar la respuesta de la pitahaya roja a la aplicación de dos abonos orgánicos sólidos en tres dosis de aplicación en las condiciones agroecológicas de San Carlos.

\section{Materiales y Métodos}

La investigación se realizó entre marzo y septiembre de 2015, que comprendió la etapa de campo (recolección de datos) en el predio "FÉNIX", el cual está ubicado en la provincia de Los Ríos, Cantón Quevedo, Parroquia San Carlos, Cantón Quevedo, Ecuador. a 79 $28^{\prime}$ de longitud oeste y $01^{\circ} 06^{\prime}$ de latitud sur y, a una altitud de 120 metros sobre el nivel del mar.

El clima se caracteriza como bosque húmedo tropical (bh-T) con topografía ligeramente plana, suelo de textura franco arcilloso, tipo Inceptisoles y $\mathrm{pH} 6$ caracterizado por una temperatura media diaria de $26,56^{\circ} \mathrm{C}$, precipitación anual promedio de $2.286 \mathrm{~mm}$, una humedad relativa $84,8 \%$ y 894 horas sol al año (División de Meteorología, Departamento de Sinóptica del Instituto Nacional Autónomo de Investigaciones Agropecuarias (INIAP), Quevedo).

\section{Material vegetativo}

En este estudio se utilizaron 315 plantas del género (Hylocereus undatus), las mismas que fueron distribuidas en tres repeticiones con un numero de 15 plantas por cada tratamiento establecido en el campo experimental.

Este género se caracteriza por ser una planta de tipo trepadora con sistema radical superficial, que puede alcanzar profundidades de hasta $15 \mathrm{~cm}$; cuyo crecimiento radicular es paralelo a la superficie del suelo. Así mismo, despliega raíces adventicias a partir de los tallos, que permiten a la planta adherirse, trepar y mantenerse erecta (Gunasena et al., 2007).

\section{Variables independientes}

Abonos orgánicos (humus y compost)

Dosis alta $8 \mathrm{tha}^{-1}$.

Dosis media $4 \mathrm{t} \mathrm{ha}^{-1}$.

Dosis baja $2 \mathrm{tha}^{-1}$.

\section{Parámetros medidos}

Número de brotes, días a floración, número de frutos, diámetro del fruto, longitud del fruto, peso de los frutos y rendimiento por ha $\mathrm{h}^{-1}$.

\section{Diseño experimental}

Para el análisis de datos, se empleó un diseño de bloques completos al azar (DBCA) con siete tratamientos y tres repeticiones. Las diferencias entre las medias de los tratamientos fueron medidas mediante el análisis de rangos múltiples de Tukey $(p \leq 0,05)$. El programa estadístico utilizado fue STAT GRAPHICS Centurión XV.

Se delimitó el área de estudio con estacas y piola identificando cada tratamiento en su respectiva repetición en el cultivo de pitahaya establecido hace tres años con 6 meses, la distancia entre plantas es de $3,0 \mathrm{~m}$ por $3.0 \mathrm{~m}$ entre hileras, dando un total de 1111 plantas ha-1. 


\section{Fertilización}

Se realizó la fertilización con la aplicación de humus y compost en tres dosis cada uno a razón de $8 ; 4$ y 2 t ha $^{-1}$, respectivamente las cuales se aplicaron directamente al suelo fraccionándolas en dos aplicaciones de 15 días para cada tratamiento (Tabla 1).

\section{Labores Culturales}

Durante la investigación se realizó varias deshierbas de forma manual (chapear) y adicionalmente se hicieron a todas las plantas una corona para mantener limpio el suelo en el contorno de cada una de ellas.

\section{Control Fitosanitario}

El control fitosanitario fue realizado mediante la aplicación preventivas de oxicloruro de cobre a una dosis de $2,5 \mathrm{~kg} \mathrm{ha}^{-1}$, que permite el control de ataque fúngico; mientras para el control de insectos se utilizó cipermetrina en dosis de $4 \mathrm{ml} \mathrm{l}^{-1}$.

Tabla 1. Distribución de los tratamientos en estudio de los abonos orgánicos.

\begin{tabular}{lc}
\hline \multicolumn{1}{c}{ Tratamientos } & Dosis t ha $^{-1}$ \\
\hline $\mathrm{T}_{1} \mathrm{AB}_{1} \mathrm{D}_{1}$ (Humus) & 8 alta \\
$\mathrm{T}_{2} \mathrm{AB}_{1} \mathrm{D}_{2}$ (Humus) & 4 media \\
$\mathrm{T}_{3} \mathrm{AB}_{1} \mathrm{D}_{3}$ (Humus) & 2 baja \\
$\mathrm{T}_{4} \mathrm{AB}_{2} \mathrm{D}_{1}$ (Compost) & 8 alta \\
$\mathrm{T}_{5} \mathrm{AB}_{2} \mathrm{D}_{2}$ (Compost) & 4 media \\
$\mathrm{T}_{6} \mathrm{AB}_{2} \mathrm{D}_{3}$ (Compost) & 2 baja \\
$\mathrm{T}_{7}$ Testigo absoluto & Sin aplicación \\
\hline
\end{tabular}

\section{Cosecha}

Esta se realizó cuando los frutos mostraron madurez, una vez cortados fueron ubicados en gavetas y posteriormente los datos respectivos fueron registrados tales como: número de frutos, diámetro del fruto, longitud del fruto y peso de los frutos.

\section{Resultados y Discusión}

\section{Número de brotes y días a la floración}

En la Tabla 2 se muestran los datos obtenidos al evaluar el número de brotes, de acuerdo al análisis de varianza se determinó que existen diferencias estadísticas significativas entre los tratamientos $(p \leq 0,05)$. Los resultados muestran que el número de brotes promedio en el testigo absoluto fue significativamente inferior al obtenido con ambos abonos y en las distintas dosificaciones empleadas; siendo los tratamientos T1 (humus) y $\mathrm{T} 4$ (compost) ambos en dosis alta $8 \mathrm{t} \mathrm{ha}^{-1}$ los que alcanzaron el mayor número de brotes promedio con 14,60 y 14,73 respectivamente; sin embargo, el número de brotes tiende a descender a medida que se disminuyen las dosis de aplicación. Al respecto Rosales et al., (2009); documentaron en sus investigaciones que Hylocereus undatus bajo fertilización orgánica alcanza 14 brotes vegetativos promedio lo cual concuerda con el resultado obtenido en esta investigación ya que le T1 (Humus en dosis alta) y T4 (Compost en dosis alta) alcanzaron 14,60 y 14,73 brotes vegetativos respectivamente.

Tabla 2. Número de brotes y días a la floración en la evaluación de dos abonos orgánicos sólidos en la producción del cultivo de pitahaya.

\begin{tabular}{llcc}
\hline & \multicolumn{1}{c}{ Tratamientos } & Numero de brotes & Días a la floración \\
\hline $\mathrm{T} 1$ & $\mathrm{AB}_{1} \mathrm{D}_{1}\left(8 \mathrm{t} \mathrm{ha}^{-1}\right.$ Humus $)$ & $14,60 \mathrm{a}$ & $19,13 \mathrm{a}$ \\
$\mathrm{T} 2$ & $\mathrm{AB}_{1} \mathrm{D}_{2}\left(4 \mathrm{t} \mathrm{ha}^{-1}\right.$ Humus $)$ & $12,60 \mathrm{~b}$ & $19,13 \mathrm{a}$ \\
$\mathrm{T} 3$ & $\mathrm{AB}_{1} \mathrm{D}_{3}\left(2 \mathrm{t} \mathrm{ha}{ }^{-1}\right.$ Humus $)$ & $10,00 \mathrm{c}$ & $19,20 \mathrm{a}$ \\
$\mathrm{T} 4$ & $\mathrm{AB}_{2} \mathrm{D}_{1}\left(8 \mathrm{t} \mathrm{ha}^{-1}\right.$ Compost $)$ & $14,73 \mathrm{a}$ & $18,93 \mathrm{a}$ \\
$\mathrm{T} 5$ & $\mathrm{AB}_{2} \mathrm{D}_{2}\left(4 \mathrm{t} \mathrm{ha}^{-1}\right.$ Compost $)$ & $12,40 \mathrm{~b}$ & $19,26 \mathrm{a}$ \\
$\mathrm{T} 6$ & $\mathrm{AB}_{2} \mathrm{D}_{3}\left(2 \mathrm{t} \mathrm{ha}{ }^{-1}\right.$ Compost $)$ & $9,60 \mathrm{c}$ & $19,73 \mathrm{a}$ \\
$\mathrm{T} 7$ & $\mathrm{Testigo} \mathrm{absoluto}_{\mathrm{C} . \text { V.\% }}$ & $7,46 \mathrm{~d}$ & $19,33 \mathrm{a}$ \\
\hline
\end{tabular}

*Letras distintas indican diferencias estadísticas significativas $(p \leq 0,05)$. 
Con respecto a los días a la floración se muestra en la Tabla 2, al realizar el análisis correspondiente se determinó que no existieron diferencias estadísticas significativas entre los tratamientos $(p \leq 0,05)$. Los promedios de los días a floración variaron entre 18,93 y 19,73 . Estos resultados se encuentran dentro del rango de floración expuesto por Le Bellec et al., (2006), quienes sostienen que en el cultivo de pitahaya desde la etapa de botón floral a floración transcurren entre 15 y 20 días, aproximadamente.

\section{Número y diámetro de los frutos a la cosecha}

La Tabla 3, muestra el número de frutos a la cosecha alcanzado en esta investigación, podemos observar que existen diferencias estadísticas significativas entre los tratamientos, el número promedio varío entre 8,13 y 13,60 frutos por tratamiento; siendo los tratamientos con dosis alta $8 \mathrm{t} \mathrm{ha}^{-1}$ (humus) y T4 (compost), los que muestra el mayor número de frutos con 13,53 y 13,60; respectivamente. Al respecto Mendoza et al., (2003) en su investigación donde evaluaron lombricompost, gallinaza, y una combinación de ambos, mencionaron que la gallinaza mostró el mejor rendimiento promedio en su investigación, sin embargo sus resultados muestran que el lombricompost en dosis alta $81 \mathrm{t} \mathrm{ha}^{-1}$ fue el segundo mejor tratamiento y no tuvo diferencias estadísticas significativas en comparación con la gallinaza cuya dosis fue $19,3 \mathrm{tha}^{-1}$, donde los rendimientos alcanzados fueron 114,7 y 132,6 $\mathrm{t} \mathrm{ha}^{-1}$, respectivamente. Así mismo, Moreira et al., (2011) sostienen que la fertilización orgánica (estiércol + gallinaza) en el cultivo de pitahaya permite obtener el mayor número de frutos promedio
6,58; siendo estadísticamente distinto al resto de los tratamientos empleados donde el granulado bioclástico mostró los resultados más bajos con 0,25 frutos a la cosecha. Estos resultados son un indicativo de que los fertilizantes orgánicos proporcionan una disponibilidad de nutrientes adecuada para las plantas, lo cual les permite incrementar su productividad.

Con respecto al diámetro de los frutos se observa en la Tabla 3, diferencias estadísticamente significativas entre los tratamientos, con diámetros que oscilaron entre 9,5 y $13,60 \mathrm{~cm}$; siendo el T1 y T4 (humus y compost) en dosis altas $8 \mathrm{t} \mathrm{ha}^{-1}$ los que registraron el mayor diámetro con $13,60 \mathrm{~cm}$ cada uno, mientras el T5 y T6 (compost) en dosis media $4 \mathrm{t} \mathrm{ha}^{-1}$ y baja $2 \mathrm{t} \mathrm{ha}^{-1}$ respectivamente muestran un comportamiento estadístico similar al testigo absoluto. En la investigación realizada por Meráz et al., (2003) y Lezama et al., (2005), sostienen que los diámetros de frutos de pitahaya pueden medir de 10 a $12 \mathrm{~cm}$, por lo tanto, los resultados obtenidos en este estudio se encuentran dentro de los rangos mencionados, siendo mejorados únicamente por el T1 y T4 que logran superar el diámetro por ellos establecidos.

\section{Longitud y peso de los frutos}

La Tabla 4, muestra la longitud promedio de los frutos se puede observar que existieron diferencias estadísticas significativas entre los tratamientos, con longitudes que oscilaron entre 7,7 y $11,8 \mathrm{~cm}$; siendo el T4 (compost) en dosis alta $8 \mathrm{t} \mathrm{ha}^{-1}$ el que registró la mayor longitud promedio con $11,8 \mathrm{~cm}$; seguido del T1 (humus) en dosis alta con 10,6 cm el cual a su vez muestra un comportamiento estadístico similar a los tratamientos T2 y T3 (humus) en

Tabla 3. Número y diámetro de frutos a la cosecha en la evaluación de dos abonos orgánicos sólidos en la producción del cultivo de pitahaya.

\begin{tabular}{clcc}
\hline & \multicolumn{1}{c}{ Tratamientos } & Número de frutos a la cosecha & Diámetro de los frutos $(\mathrm{cm})$ \\
\hline $\mathrm{T} 1$ & $\mathrm{AB}_{1} \mathrm{D}_{1}\left(8 \mathrm{t} \mathrm{ha}^{-1}\right.$ Humus $)$ & $13,53 \mathrm{a}$ & $13,6 \mathrm{a}$ \\
$\mathrm{T} 2$ & $\mathrm{AB}_{1} \mathrm{D}_{2}\left(4 \mathrm{t} \mathrm{ha}^{-1}\right.$ Humus $)$ & $11,40 \mathrm{~b}$ & $11,0 \mathrm{~b}$ \\
$\mathrm{~T} 3$ & $\mathrm{AB}_{1} \mathrm{D}_{3}\left(2 \mathrm{t} \mathrm{ha}^{-1}\right.$ Humus $)$ & $9,66 \mathrm{~cd}$ & $10,0 \mathrm{bc}$ \\
$\mathrm{T} 4$ & $\mathrm{AB}_{2} \mathrm{D}_{1}\left(8 \mathrm{t} \mathrm{ha}^{-1}\right.$ Compost $)$ & $13,60 \mathrm{a}$ & $13,6 \mathrm{a}$ \\
$\mathrm{T} 5$ & $\mathrm{AB}_{2} \mathrm{D}_{2}\left(4 \mathrm{t} \mathrm{ha}^{-1}\right.$ Compost $)$ & $10,93 \mathrm{bc}$ & $9,9 \mathrm{c}$ \\
$\mathrm{T} 6$ & $\mathrm{AB}_{2} \mathrm{D}_{3}\left(2 \mathrm{t} \mathrm{ha}^{-1}\right.$ Compost $)$ & $9,80 \mathrm{c}$ & $9,3 \mathrm{c}$ \\
$\mathrm{T} 7$ & Testigo absoluto & $8,13 \mathrm{~d}$ & $9,5 \mathrm{c}$ \\
\hline & C.V.\% & 17,82 & 16,19 \\
\hline
\end{tabular}

*Letras distintas indican diferencias estadísticas significativas $(p \leq 0,05)$. 
Tabla 4. Longitud y peso de los frutos en la evaluación de dos abonos orgánicos sólidos en la producción del cultivo de pitahaya.

\begin{tabular}{llcc}
\hline & \multicolumn{1}{c}{ Tratamientos } & Longitud de los frutos $(\mathrm{cm})$ & Peso de los frutos \\
\hline $\mathrm{T} 1$ & $\mathrm{AB}_{1} \mathrm{D}_{1}\left(8 \mathrm{tha}^{-1}\right.$ Humus $)$ & $10,6 \mathrm{~b}$ & $0,733 \mathrm{a}$ \\
$\mathrm{T} 2$ & $\mathrm{AB}_{1} \mathrm{D}_{2}\left(4 \mathrm{tha}^{-1}\right.$ Humus $)$ & $10,2 \mathrm{bc}$ & $0,726 \mathrm{a}$ \\
$\mathrm{T} 3$ & $\mathrm{AB}_{1} \mathrm{D}_{3}\left(2 \mathrm{t} \mathrm{ha}^{-1}\right.$ Humus $)$ & $9,6 \mathrm{bc}$ & $0,704 \mathrm{a}$ \\
$\mathrm{T} 4$ & $\mathrm{AB}_{2} \mathrm{D}_{1}\left(8 \mathrm{tha}^{-1}\right.$ Compost $)$ & $11,8 \mathrm{a}$ & $0,743 \mathrm{a}$ \\
$\mathrm{T} 5$ & $\mathrm{AB}_{2} \mathrm{D}_{2}\left(4 \mathrm{t} \mathrm{ha}^{-1}\right.$ Compost $)$ & $10,0 \mathrm{bc}$ & $0,674 \mathrm{a}$ \\
$\mathrm{T} 6$ & $\mathrm{AB}_{2} \mathrm{D}_{3}\left(2 \mathrm{t} \mathrm{ha}^{-1}\right.$ Compost $)$ & $9,5 \mathrm{c}$ & $0,668 \mathrm{a}$ \\
$\mathrm{T} 7$ & Testigo absoluto & $7,7 \mathrm{~d}$ & $0,523 \mathrm{~b}$ \\
\hline C.V.\% & 12,08 & 10,93 \\
\hline
\end{tabular}

*Letras distintas indican diferencias estadísticas significativas $(p \leq 0,05)$.

dosis media $4 \mathrm{t} \mathrm{ha}^{-1}$ y baja $2 \mathrm{t} \mathrm{ha}^{-1}$ con 10,2 y 9 , $6 \mathrm{~cm}$; respectivamente. Esto concuerdan con los resultados obtenidos por Castillo et al., (2005) quienes en la caracterización morfológica de cinco genotipos de pitahaya (Hylocereus undatus), donde cuatro correspondían a genotipos con pericarpio rojizo determinaron que la longitud del fruto en los cuatro genotipos varía desde 7,4 hasta 9,6 cm. Así mismo López y Guido (2002); en su ficha técnica manifiestan que la longitud del fruto puede variar entre $8 \mathrm{~cm}$ y $12 \mathrm{~cm}$, lo cual se ajusta a los datos obtenidos en esta investigación.

En relación al peso de frutos se observa en la (Tabla 4), el peso promedio de los frutos de Hylocereus undatus (pitahaya) obtenidos en esta investigación, de acuerdo al análisis realizado se determinó que no existen diferencias estadísticas significativas entre los tratamientos con fertilización orgánica; sin embargo estos si muestran diferencias significativas con respecto al T7 (testigo absoluto) que mostró el menor peso promedio alcanzado con $0,523 \mathrm{~kg}$; siendo los T1 y T4 (humus y compost) en dosis alta $8 \mathrm{t} \mathrm{ha}^{-1}$ los que registraron los pesos promedios más altos con 0,733 y $0,743 \mathrm{~kg}$; respectivamente. Se pudo observar además que, aunque no existieron diferencias estadísticamente significativas entre los tratamientos con fertilización orgánica fueron el T5 y T6 (compost) en dosis media $4 \mathrm{t} \mathrm{ha}^{-1}$ y baja $2 \mathrm{t} \mathrm{ha}^{-1}$, los que mostraron los pesos de frutos más bajos de los tratamientos orgánicos con 0,674 y 0,668 kg. Estos datos superan a lo documentado por Sotelo et al., (2005) quienes sostienen que la pitahaya (Hylocereus undatus), alcanza un peso promedio de $0,370 \mathrm{~kg}$. Así como, a los registrados por Castillo et al., (2005) quienes manifiestan que en genotipos de pitahaya (Hylocereus undatus) de pericarpio rojizo el peso del fruto puede variar de 0,212 a $0,436 \mathrm{~kg}$.

\section{Rendimiento por hectárea}

En la Tabla 5, se observa el rendimiento promedio por hectárea en el cultivo de pitahaya,

Tabla 5. Rendimiento por hectárea en la evaluación de dos abonos orgánicos sólidos en la producción del cultivo de pitahaya.

\begin{tabular}{llc}
\hline & \multicolumn{1}{c}{ Tratamientos } & Rendimiento por hectárea $(\mathrm{kg})$ \\
\hline $\mathrm{T} 1$ & $\mathrm{AB}_{1} \mathrm{D}_{1}\left(8 \mathrm{t} \mathrm{ha}^{-1}\right.$ Humus $)$ & $11021,10 \mathrm{a}^{*}$ \\
$\mathrm{~T} 2$ & $\mathrm{AB}_{1} \mathrm{D}_{2}\left(4 \mathrm{t} \mathrm{ha}^{-1}\right.$ Humus $)$ & $9202,78 \mathrm{~b}$ \\
$\mathrm{~T} 3$ & $\mathrm{AB}_{1} \mathrm{D}_{3}\left(2 \mathrm{t} \mathrm{ha}^{-1}\right.$ Humus $)$ & $7568,28 \mathrm{c}$ \\
$\mathrm{T} 4$ & $\mathrm{AB}_{2} \mathrm{D}_{1}\left(8 \mathrm{t} \mathrm{ha}^{-1}\right.$ Compost $)$ & $11229,20 \mathrm{a}$ \\
$\mathrm{T} 5$ & $\mathrm{AB}_{2} \mathrm{D}_{2}\left(4 \mathrm{t} \mathrm{ha}^{-1}\right.$ Compost $)$ & $727,70 \mathrm{bc}$ \\
$\mathrm{T} 6$ & $\mathrm{AB}_{2} \mathrm{D}_{3}\left(2 \mathrm{t} \mathrm{ha}^{-1}\right.$ Compost $)$ & $4725,45 \mathrm{c}$ \\
$\mathrm{T} 7$ & $\mathrm{Testigo} \mathrm{absoluto}^{\text {C.V. }(\%)}$ & 25,69 \\
\hline
\end{tabular}

*Letras distintas indican diferencias estadísticas significativas $(p \leq 0,05)$. 
se observaron diferencias estadísticamente significativas entre los tratamientos con rendimientos que oscilan entre 4725,45 y $11229,20 \mathrm{~kg} \mathrm{ha}^{-1}$; siendo los tratamientos T1 y $\mathrm{T} 4$ (humus y compost) en dosis altas $8 \mathrm{tha}^{-1}$ los que lograron alcanzar los máximos rendimientos en este estudio con 11021,10 y 11229,20 kg ha-1; respectivamente, seguidos por los tratamientos que comprenden humus y compost en dosis media $4 \mathrm{t} \mathrm{ha}^{-1}$ y baja $2 \mathrm{t} \mathrm{ha}^{-1}$ los cuales disminuyen su rendimiento a medida que decrece la dosis. Al comparar entre los abonos se determinó que ambos muestran un comportamiento estadístico similar cuando son evaluados a dosis iguales. Estos resultados concuerdan con los reportados con Jordán et al., (2005) quienes manifiestan que la pitahaya roja puede alcanzar de 8000 a 10000 $\mathrm{kg} \mathrm{ha}^{-1}$. Sin embargo, discrepan con los resultados obtenidos por López y Miranda (1998) quienes en su investigación utilizaron fertilización mineral lograron obtener rendimientos entre 1613,91 y $6730,54 \mathrm{~kg} \mathrm{ha}^{-1}$.

\section{Conclusiones}

Los abonos orgánicos (humus y compost) utilizados en este experimento para la fertilización del cultivo de pitahaya roja (Hylocereus undatus), mostraron un efecto similar cuando fueron utilizados en las mismas dosis; cada una de las variables evaluadas tuvo una tendencia a la disminución a medida que decreció la dosis de abono aplicada.

Ambos abonos mostraron la capacidad de incrementar los rendimientos en el cultivo de pitahaya roja (Hylocereus undatus), con respecto al testigo absoluto; el rendimiento estuvo en relación directa con la producción de frutos por planta.

La dosis que permitió maximizar el rendimiento en el cultivo de pitahaya roja (Hylocereus undatus) en ambos tipos de abono orgánico fue la de $8 \mathrm{tha}^{-1}$.

\section{Literatura Citada}

Altieri, M.; Rosset, P.

1995. Agroecology and the conversion of large-scale conventional systems to sustainable management. International Journal of Environmental Studies, 50: 165-185.

Castillo, R.; Livera, M.; Márquez, G.

2005. Caracterización morfológica y compatibilidad sexual de cinco genotipos de pitahaya. Agrociencia, 39 (2): 183-194.

Gunasena, H.; Pushpakumara, D.; Kariyawasam, M.

2007. Dragon fruit, Hylocereus undatus (Haw.) Britton and Rose. Chapter 4. En: Pushpakumara, D.K.N.G.; Gunasena, H.P.M.; Sing, V.P. (Eds.). Underutilized fruit trees in Sri Lanka. World Agroforestry Centre. New Delhi, India. Pp. 110-142.

Iguácel, F.; Rosa, M.; Orús, F.; Quílez, D.

2010. Fertilización con purín en doble cultivo anual, en mínimo laboreo, y riego por aspersión. Informaciones Técnicas del Departamento de Agricultura y Alimentación del Gobierno de Aragón. España. 12 p.

Jordán, M.; Vásconez, S.; Véliz, D.; González, V.

2005. Cracterización morfológica y compatibilidad sexual de cinco genotipos de pitahaya (Hylocereus undatus). Agrociencia, 39: 183-194.

Le Bellec, F.; Vaillant, F.; Imbert, E.

2006. Pitahaya (Hylocereus spp): a new fruitcrop, a marketwith a future. Fruits, 61: 237-250.

Lezama, A.; Tapia, A.; Miñoz, G.; Zepeda, B.

2005. El cultivo de la Pitahaya. sistema de agronegocios de traspatio. Secretaria de agricultura y ganaderia, desarrollo rural pezca y alimentacion. Subsecretaria de desarrollo rular dirección nacional de apoyo para el desarrollo rural. Puebla, Mexico. 11 p.

López, H.; Guido, A.; Menocal, O.; Castillo, G. 2002. Guia técnologica 6. Cultivo de la pitahaya. Instituto Nicaraguense de Tecnología. Manuagua, Nicaragua. 28 p.
López, H.; Miranda, A.

2002. Cultivo de la Pitahaya. Guía Tecnológica 6. Instituto Nicaragüense de Tecnología Agropecuaria. INTA 38 p.

López, O.; Miranda, A.

1998. Evaluación de dosis de nitrógeno y fósforo en el cultivo de pitahaya (Hylocereus undatus). Agronomía Mesoamericana, 9 (1): 66-71.

Manani, P.; Chávez, E.; Ortuño, N.

2012. Biofertilizante casero para la producción ecologica de cultivos. Editorial. Bogota, Colombia 11 p.

Mendoza, H.; Carrillo, J.; Perales, C.; Ruiz, J.

2003. Evaluación de fuentes de fertilización orgánica para tomate de invernadero en Oaxaca, México. Revista Manejo Integrado de Plagas y Agroecología, Costa Rica, 70: 30-35.

Meráz, M.; Gómez, M.; Schwentesius, R.

2003. Pitayas y Pitahayas. En: Flores Valez, C.A. (Ed.). Pitahaya de México - Producción y comercialización en el contexto internacional. Universidad Autónoma Chapingo. Chapingo, Mexico 173 p.

Montesinos, J.; Rodríguez, L.; Ortiz, R.; Fonseca, M.; Ruíz, G.; Guevara, F.

2015. Pitahaya. (Hylocereus spp.) un recurso filogenético con historia y futuro para el trópico seco mexicano. Review. Ministerio de Educación Superior. Cuba Instituto Nacional de Ciencias Agrícolas. Cultivos Tropicales, 36 ( $\mathrm{N}^{\mathrm{o}}$ Especial): 67-76.

Moreira, A.; Ramos, J.; Araújo, A.; Marques, B.

2011. Produção e qualidade de frutos de pitaia-vermelha com adubação orgânica e granulado bioclástico. Revista Brasileira de Fruticultura, 33: 762-766.

Organización de las Naciones Unidas para la Agricultura y la Alimentación.

1999. Guía para el manejo eficiente de la nutrición de las plantas. FAO. Roma, Italia. $20 \mathrm{p}$. 
Otiniano, J.; Meneses, L.; Blas, R.; Bello, A.

2006. La materia orgánica, importancia y experiencia de su uso en la agricultura. Idesia (Arica), 24 (1): 49-61.

Ponce, A.; kodera, Y.

2003. Evacuación de dosis de fertilizantes fuentes de fertilización orgánica en pitahaya (Hylocereus undatus). INTA. Managua, Nicaragua. $10 \mathrm{p}$.
Rosales, E.; Luna, C.; Cruz, A.

2009. Clasificación y selección tradicional de Pitahaya (Stenocereus Pruinosus (Otto) Buxb) en Tianguistengo, Oaxaca Y variación morfológica de cultivares. Revista Chapingo Serie Horticultura, 15 (1): 75-82.

Sotelo, E.; Ortiz, C.; Rizo, M.

2005. Áreas potenciales para el cultivo de pitahaya (Hylocereus undatus (Haw) Britt \& Rose), en el sur del Estado de México. Ciencia Forestal en México, 30 (98): 87-97. 
